\title{
TWO GENERALIZATIONS OF THE GLEASON-KAHANE-ŻELAZKO THEOREM
}

\author{
ERIK Christensen
}

In this article we obtain 2 generalizations of the well known Gleason-Kahane-Żelazko Theorem. We consider a unital Banach algebra $\mathfrak{A}$, and a continuous unital linear mapping $\varphi$ of $\mathfrak{A}$ into $M_{n}(\mathbb{C})$ - the $n \times n$ matrices over $\mathbb{C}$. The first generalization states that if $\varphi$ sends invertible elements to invertible elements, then the kernel of $\varphi$ is contained in a proper two sided closed ideal of finite codimension. The second result characterizes this property for $\varphi$ in saying that $\varphi\left(\mathfrak{A}_{\text {inv }}\right)$ is contained in $\mathrm{GL}_{n}(\mathbb{C})$ if and only if for each $a$ in $\mathfrak{A}$ and each natural number $k$ :

$$
\operatorname{trace}\left(\varphi\left(a^{k}\right)\right)=\operatorname{trace}\left(\varphi(a)^{k}\right) .
$$

\section{Introduction.}

The results are based on Aupetits work [1], where he proves that if $\varphi$ is surjective and $\varphi\left(\mathfrak{A}_{\text {inv }}\right)$ is contained in $\mathrm{GL}_{n}(\mathbb{C})$ then $\varphi$ is a Jordan homomorphism. Some of the key ingredients in Aupetits proof are some relations which involve $\varphi$ and the trace. We quote $[1, \operatorname{Rel}(3)$, p. 15]

$$
\operatorname{trace}(\varphi(x y))=\operatorname{trace}(\varphi(x) \varphi(y)) .
$$

The results in this paper are based on some elaboration of the identity above.

Aupetits result is proved via a Liouville Theorem for harmonic functions. Since we could not get the reference quoted in [1] for this result, we have included a slightly different argument, which on the other hand is an application of the Hadamard Factorization Theorem, very much in the same way as used in $[3,4]$.

\section{Notation and basic results.}

We will consider a unital Banach algebra $\mathfrak{A}$ over the complex numbers, and let $\mathfrak{A}_{\text {inv }}$ denote the set of invertible elements in $\mathfrak{A}$. For a natural number $n$ we let $M_{n}(\mathbb{C})$ denote the $n \times n$ matrices over $\mathbb{C}$ and we will let $\operatorname{tr}(\cdot)$ denote the usual trace on $M_{n}(\mathbb{C})$, which satisfies $\operatorname{tr}(I)=n$. We remind the reader that a functional $f$ on an algebra $\mathfrak{B}$ is called a trace if $f(a b)=f(b a)$ for all 
$a, b$ from $\mathfrak{B}$. Moreover - up to scalar multiples - there exists only one trace on $M_{n}(\mathbb{C})$.

The following lemma is well known, but we do not have an exact reference.

Lemma 2.1. There exists a positive real $r(0<r<1)$ such that for all $x$ in $M_{n}(\mathbb{C})$ with $\|x-I\|<r$ we have

$$
|\operatorname{det}(x)-1|<1 \quad \text { and } \quad \log (\operatorname{det}(x))=\operatorname{tr}(\log (x)) .
$$

Proof. The existence of $r(0<r<1)$ such that $\|x-I\|<r \Rightarrow|\operatorname{det}(x)-1|<1$ follows from the continuity of the determinant. Suppose now $x$ in $M_{n}(\mathbb{C})$ is chosen such that $\|x-I\|<r<1$ then the power series for $\log (1+u)$ in the circle $\{u \in \mathbb{C}|| u \mid<1\}$ converges for both $(\operatorname{det}(x)-1)$ and $(x-I)$ so the expressions make sense. The equality is easily obtained when $x$ is represented in a Jordan normal form.

\section{Main results.}

We start by recapturing the basic results from [1] in Theorem 3.1, and then we present our extensions.

Theorem 3.1. Let $\mathfrak{A}$ be a unital Banach algebra $\varphi$ a continuous unital linear mapping of $\mathfrak{A}$ into $M_{n}(\mathbb{C})$.

If $\varphi\left(\mathfrak{A}_{\text {inv }}\right) \subseteq \mathrm{GL}_{n}(\mathbb{C})$ then:

(i) $\forall a, b \in \mathfrak{A}: \operatorname{det}\left(\varphi\left(e^{a} e^{b}\right)\right)=\operatorname{det}\left(e^{\varphi(a)} e^{\varphi(b)}\right)$,

(ii) $\forall a, b \in \mathfrak{A}: \operatorname{tr}(\varphi(a b))=\operatorname{tr}(\varphi(a) \varphi(b))=\operatorname{tr}(\varphi(b a))$,

(iii) $\forall a, b \in \mathfrak{A}: \operatorname{det}(\varphi(a b))=\operatorname{det}(\varphi(a) \varphi(b))$.

Proof. For an $a$ in $\mathfrak{A}$ we define an entire function $f(z)$ by

$$
f(z)=\operatorname{det}\left(\varphi\left(e^{z a}\right) e^{-z \varphi(a)}\right) .
$$

As usual $f(z) \neq 0$ for all $z$ and the order $p$ of $f$ satisfies $p \leq 1$ since

$$
|f(z)| \leq\|\varphi\|^{n} \exp (|z| n(\|a\|+\|\varphi(a)\|)) .
$$

By Hadamards Factorization Theorem [2, p. 291; 5 p. 250] we have $f(z)=$ $e^{\alpha+\beta z}$, but $f(0)=1$ so $f(z)=e^{\beta z}$.

Following Lemma 2.1 we get that for some positive real $r$ we have

$$
\begin{aligned}
\forall z,|z|<r: \beta z & =\operatorname{tr}\left(\log \left(I+z \varphi(a)+O\left(z^{2}\right)\right)\right)-z \operatorname{tr}(\varphi(a)) \\
& =O\left(z^{2}\right) .
\end{aligned}
$$


Hence $\beta=0$ and $\operatorname{det}\left(\varphi\left(e^{a}\right)\right)=\operatorname{det}\left(e^{\varphi(a)}\right)$.

In the general case define an entire function $f(w, z)$ in 2 variables by $f(w, z)=\operatorname{det}\left(\varphi\left(e^{w a} e^{z b}\right) e^{-w \varphi(a)} e^{-z \varphi(b)}\right)$. Let $w$ be fixed then the function $g(z)=f(w, z)$ is entire, never vanishing, of order 1 and by the previous result $g(0)=1$, hence there exists a complex function $\alpha(w)$ such that $f(w, z)=g(z)=e^{\alpha(w) z}$. By analogy we find a complex function $\beta(z)$ such that $f(w, z)=e^{w \beta(z)}$. Hence there exists a constant $\gamma$ such that $f(w, z)=e^{\gamma w z}$. On the other hand the function $k(z)=f(z, z)$ is easily seen to be of order less than or equal to 1 so $\gamma=0$ and (i) follows.

Applying Lemma 2.1 to both sides in the following identity

$$
\operatorname{det}\left(e^{w \varphi(a)}\right) \operatorname{det}\left(e^{z \varphi(b)}\right)=\operatorname{det}\left(\varphi\left(e^{w a} e^{z b}\right)\right)
$$

shows that there exists a positive real $r$ such that for all $z, w$ in $\mathbb{C}$ with $|z|<r,|w|<r$

$$
\begin{aligned}
w & \operatorname{tr}(\varphi(a))+z \operatorname{tr}(\varphi(b))=\operatorname{tr}\left(\log \left(\varphi\left(e^{w a} e^{z b}\right)\right)\right) \\
& =\operatorname{tr}\left(\log \left(I+w \varphi(a)+z \varphi(b)+w z \varphi(a b)+w^{2} p_{1}(w, z)+z^{2} p_{2}(w, z)\right)\right) \\
& =w \operatorname{tr}(\varphi(a))+z \operatorname{tr}(\varphi(b))+w z \operatorname{tr}(\varphi(a b)) \\
& -\frac{1}{2} w z \operatorname{tr}(\varphi(a) \varphi(b)+\varphi(b) \varphi(a))+w^{2} p_{3}(w, z)+z^{2} p_{4}(w, z)
\end{aligned}
$$

where $p_{i}(w, z)$ are power series. Hence (ii) follows from the properties of the trace. The relation (iii) is a consequence of (i) since for $|z|>\|a\|+\|b\|$ we have $(z-a)=z\left(1-\frac{a}{z}\right)=z \exp \left(\log \left(I-\frac{a}{z}\right)\right)$ and a similar expression for $b$ and hence for $|z|>\|a\|+\|b\|$ we have

$$
\operatorname{det}(\varphi((a-z)(b-z)))=\operatorname{det}(\varphi(a-z)) \operatorname{det}(\varphi(b-z)) .
$$

Since the functions involved are entire, we get (iii) for $z=0$.

The relation (ii) is the basis for the following result.

Theorem 3.2. Let $\mathfrak{A}$ be a unital Banach algebra and $\varphi$ a continuous, unital linear mapping of $\mathfrak{A}$ into $M_{n}(\mathbb{C})$.

If $\varphi\left(\mathfrak{A}_{\text {inv }}\right) \subseteq \mathrm{GL}_{n}(\mathbb{C})$ then $\mathfrak{A}$ has a proper closed two sided ideal $J$ - of finite codimension - which contains the kernel of $\varphi$.

Proof. Define $J=\{a \in \mathfrak{A} \mid \forall b \in \mathfrak{A}: \operatorname{tr}(\varphi(a b))=0\}$, then $J$ is obviously a closed right ideal, but by the trace property $-\operatorname{tr}(\varphi(a b))=\operatorname{tr}(\varphi(b a))-$ from (ii) in Theorem 3.1 we see that $J$ is a left ideal as well. The property $\operatorname{tr}(\varphi(a b))=\operatorname{tr}(\varphi(a) \varphi(b))$ from (ii) above shows that $\operatorname{ker} \varphi \subseteq J$. Hence $J$ is 
of finite codimension and since $\varphi(I)=I$ we get $I \notin J$ and $J$ is a proper ideal.

Corollary 3.3. Let $k, n$ be natural numbers if $\varphi$ is a unital linear mapping of $M_{k}(\mathbb{C})$ into $M_{n}(\mathbb{C})$ which satisfy $\varphi\left(\mathrm{GL}_{k}(\mathbb{C})\right) \subseteq \mathrm{GL}_{n}(\mathbb{C})$, then $\varphi$ is injective and $k$ divides $n$.

Proof. Since $M_{k}$ has no nontrivial ideals, we get $\operatorname{ker} \varphi=\{0\}$ and $\varphi$ is injective. By (ii) in Theorem 3.1 we get that $\operatorname{tr}_{n} \circ \varphi$ is a trace on $M_{k}(\mathbb{C})$ which satisfies $\operatorname{tr}_{n}(\varphi(I))=\operatorname{tr}_{n}(I)=n$. By the uniqueness (up to scalar multiples) of the trace on $M_{k}(\mathbb{C})$ we have for all $a$ in $M_{k}(\mathbb{C}): \operatorname{tr}_{n}(\varphi(a))=(n / k) \operatorname{tr}_{k}(a)$. Let $e$ be a rank 1 projection in $M_{k}(\mathbb{C})$ then $\sigma(\varphi(e)) \subseteq \sigma(e)=\{0,1\}$ so $\operatorname{tr}_{n}(\varphi(e)) \in \mathbb{N}_{0}$. On the other hand $\operatorname{tr}_{n}(\varphi(e))=(n / k) \operatorname{tr}_{k}(e)=n / k$ so $n / k \in \mathbb{N}$.

Corollary 3.4. If $\varphi\left(\mathfrak{A}_{\mathrm{inv}}\right) \subseteq \mathrm{GL}_{n}(\mathbb{C})$ then there exists a unital finitedimensional algebra $\mathfrak{B}$ and a linear mapping $\psi$ of $M_{n}(\mathbb{C})$ into $\mathfrak{B}$ such that $\psi \circ \varphi$ is a unital homomorphism.

Proof. Just another formulation of the result $\operatorname{ker} \varphi \subseteq J \neq \mathfrak{A}$, combined with elementary algebra.

The property (ii) from Theorem 3.1 and the theorem above are generalizations of the original Gleason-Kahane-Żelazko Theorem. The following result yields another generalisation as well as a characterization of the mappings $\varphi$ which satisfy $\varphi\left(\mathfrak{A}_{\text {inv }}\right) \subseteq \mathrm{GL}_{n}(\mathbb{C})$.

Theorem 3.5. Let $\mathfrak{A}$ be a unital Banach algebra and $\varphi$ a unital continuous linear mapping into $M_{n}(\mathbb{C})$. Then $\varphi\left(\mathfrak{A}_{\mathrm{inv}}\right) \subseteq \mathrm{GL}_{n}(\mathbb{C})$ if and only if

$$
\forall k \in \mathbb{N} \forall a \in \mathfrak{A}: \operatorname{tr}\left(\varphi\left(a^{k}\right)\right)=\operatorname{tr}\left(\varphi(a)^{k}\right) .
$$

Proof. Suppose first that $\varphi\left(\mathfrak{A}_{\text {inv }}\right) \subseteq \mathrm{GL}_{n}(\mathbb{C})$. Let $r$ be the positive real comming from Lemma 2.1 and let $a$ be in $\mathfrak{A}$ and $z$ in $\mathbb{C}$, then there exists a positive real $r_{1}, 0<r_{1}<\|a\|^{-1}$ such that for $|z|<r_{1}$

$$
\|\varphi(I+z a)-I\|<r \text { and }\|\exp (\varphi(\log (I+z a)))-I\|<r .
$$

By Theorem 3.1 (i) we get for $|z|<r_{1}$

$$
\operatorname{det}(\varphi(\exp (\log (I+z a))))=\operatorname{det}(\exp (\varphi(\log (I+z a))))
$$


so by Lemma 2.1

$$
\forall z,|z|<r_{1}: \operatorname{tr}(\log (I+z \varphi(a)))=\operatorname{tr}(\varphi(\log (1+z a))) .
$$

By expanding in power series and comparing terms we get

$$
\forall k \in \mathbb{N}: \quad(-1)^{k-1} k^{-1} \operatorname{tr}\left(\varphi(a)^{k}\right)=(-1)^{k-1} k^{-1} \operatorname{tr}\left(\varphi\left(a^{k}\right)\right),
$$

and the first part of the proof is complete. Let us now suppose, that for each $k$ in $\mathbb{N}$ and any $a$ in $\mathfrak{A} \operatorname{tr}\left(\varphi\left(a^{k}\right)\right)=\operatorname{tr}\left(\varphi(a)^{k}\right)$. Let $b$ be an element in $\mathfrak{A}$ and let $\mathfrak{C}$ denote the abelian unital Banach algebra generated by $b$ and all its resolvents in $\mathfrak{A}$. Since $\mathfrak{C}$ is abelian we get for $c, d$ in $\mathfrak{C} c d=\frac{1}{4}\left((c+d)^{2}-(c-d)^{2}\right)$ so

$(*) \operatorname{tr}(\varphi(c d))=\frac{1}{4}\left(\operatorname{tr}\left((\varphi(c)+\varphi(d))^{2}-(\varphi(c)-\varphi(d))^{2}\right)\right)=\operatorname{tr}(\varphi(c) \varphi(d))$.

Having this identity we may as in the proof of Theorem 3.2 define a two sided ideal $J_{\mathfrak{C}}$ in $\mathfrak{C}$ by

$$
J_{\mathfrak{C}}=\{c \in \mathfrak{C} \mid \forall d \in \mathfrak{C}: \operatorname{tr}(\varphi(c d))=0\} .
$$

Again $J_{\mathfrak{C}} \neq \mathfrak{C}$ and

$$
(\operatorname{ker} \varphi \cap \mathfrak{C}) \subseteq J_{\mathfrak{C}}
$$

Since $J_{\mathfrak{C}}$ is a proper two sided ideal in $\mathfrak{C}$ and $b$ is invertible in $\mathfrak{C}, b \notin J_{\mathfrak{C}}$, and $\varphi(b) \neq 0$. Let $p(x)$ be a monic polynomial which satisfies $p(\varphi(b))=0$. The roots for $p$ are divided into two groups $L=\left\{\lambda_{1}, \ldots, \lambda_{k}\right\}$ and $M=$ $\left\{\mu_{1}, \ldots, \mu_{l}\right\}$ corresponding to the criteria: for each $\lambda_{i}$ we have $\left(b-\lambda_{i}\right)$ is not invertible in $\mathfrak{C}$ and for each $\mu_{j},\left(b-\mu_{j}\right)$ is invertible in $\mathfrak{C}$. Finally, there exist exponents $r_{1}, \ldots, r_{k}$ and $s_{1}, \ldots, s_{l}$ such that

$$
p(x)=\left(\prod_{i=1}^{k}\left(x-\lambda_{i}\right)^{r_{i}}\right)\left(\prod_{j=1}^{l}\left(x-\mu_{j}\right)^{s_{j}}\right) .
$$

In order to link properties of $p(b)$ to properties of $p(\varphi(b))=0$ we state and prove that for any polynomial $q$ and any $c, d$ in $\mathfrak{C}$ we have

$$
\operatorname{tr}(\varphi(q(c) d))=\operatorname{tr}(\varphi(q(c)) \varphi(d))=\operatorname{tr}(q(\varphi(c)) \varphi(d)) .
$$

The proof of $(* *)$ follows from the proof of the special case where $q(x)=$ $x^{s}, s \in \mathbb{N}$. Let $z \in \mathbb{C}$ then by assumption

$$
\forall z \in \mathbb{C}: \quad \operatorname{tr}\left(\varphi\left((c+z d)^{s+1}\right)\right)=\operatorname{tr}\left((\varphi(c)+z \varphi(d))^{s+1}\right)
$$


and then by comparing terms

$$
\operatorname{tr}\left(\varphi\left(c^{s} d\right)\right)=\operatorname{tr}\left(\varphi(c)^{s} \varphi(d)\right) .
$$

Having $(* *)$ we get $p(b) \in J_{\mathfrak{C}}$. Since the elements $\left(b-\mu_{j}\right)$ are invertible in $\mathfrak{C}$ we get for the polynomial $q(x)=\prod_{i=1}^{k}\left(x-\lambda_{i}\right)^{r_{i}}$ that $q(b) \in J_{\mathfrak{C}}$. By definition of $J_{\mathfrak{C}}$ and by $(* *)$ we then have

$$
\forall s \in \mathbb{N}: \quad \operatorname{tr}\left((q(\varphi(b)))^{s}\right)=\operatorname{tr}\left(\varphi\left(q(b)^{s}\right)\right)=0 .
$$

The matrix $q(\varphi(b))$ is then nilpotent and then for each $\lambda$ in $\sigma(\varphi(b))$ there exists an $i \in\{1, \ldots k\}$ such that $\lambda=\lambda_{i} \in \sigma(b)$, and we have proved that $\sigma(\varphi(b)) \subseteq \sigma(b)$, so $\varphi\left(\mathfrak{A}_{\text {inv }}\right) \subseteq \mathrm{GL}_{n}(\mathbb{C})$.

\section{References}

[1] B. Aupetit, Une generalisation du théorème de Gleason-Kahane-Żelazko pour les algèbres de Banach, Pac. J. Math., 85 (1979), 11-17.

[2] J.B. Conway, Functions of one complex variable, Grad. Texts in Math., Springer, 111975.

[3] A.M. Gleason, A characterization of maximal ideals, J. Analyse Math., 19 (1967), 171-172.

[4] J.P. Kahane and W. Żelazko, A characterization of maximal ideals in commutative Banach algebras, Studia Math., 29 (1968), 339-343.

[5] E.C. Titchmarsh, The theory of functions, The Clarendon Press, 1932.

Received April 19, 1995 and revised September 18, 1995.

K申BENHAVNS UNIVERSITET

UNIVERSITETSPARKEN 5

DK-2100 Copenhagen $\emptyset$, Denmark 